\title{
Herpetic Keratitis Recurred after the Use of a Thermal Medical Device: A Case Report
}

\section{Dear Editor,}

Herpetic keratitis is a relatively common disease known to respond well to treatment, but relapses are common. Numerous studies have reported the incidence is $0.01 \%$ to $0.02 \%$ with recurrence rates estimated to be $40 \%$ to $50 \%$ within 5 years [1]. Herpes simplex virus (HSV) replicates itself to infect the sensory axons of the cornea and infiltrate to the neurons inside the trigeminal ganglia. Even if treatment is completed, the ganglion is subjected to a series of steps to activate the transcription substance of the viral genome for reactivation immediately [1]. Because the recurrence is associated with poor visual prognosis, much effort is required to prevent recurrence. In recent studies, heat stress has been known to influence on reactivation of HSV [2]. Thermal therapy became popular as a treatment for meibomian gland dysfunction and dry eye syndrome, but studies of side effects are lacking. We try to report a rare case of herpetic keratitis recurred after the use of a thermal medical device.

A 67-year-old female patient presented with foreign body sensation and decreased visual acuity in her right eye for several days. The patient had no medical history except hypertension. She had been diagnosed with herpetic keratitis 5 years ago for the first time and had histories of recurrent herpetic keratitis 1 year and 6 months ago. Visual acuity of right eye was $20 / 800$ and the intraocular pressure was $10 \mathrm{mmHg}$. A slit lamp examination showed a dendrite lesions stained with fluorescein, corneal edema, stromal infiltration, and keratic precipitates (Fig. 1A). There were no inflammatory cells in the anterior chamber and no abnormal findings on fundus examination. The pa-

Received: January 13, 2021 Final revision: November 18, 2021

Accepted: November 22, 2021 tient told symptoms had been worse since taking a thermal medical device (Rapha402; Rapha Medical, Seoul, Korea) every morning and night for 5 minutes each, for 10 days. The device was applied directly on the posterior portion of eyelid and direct contact to ocular surface was not happened. The temperature range of the device was from $36^{\circ} \mathrm{C}$ to $45^{\circ} \mathrm{C}$. As no definite reason affecting recurrence was observed other than this thermal device, we diagnosed as recurrent herpetic keratitis caused by heat stress and treated with topical ganciclovir $0.15 \%$. After confirming corneal epithelial lesion improvement, the patient was treated with oral methylprednisolone with tapering to minimize corneal opacity. After 3 months of topical antiviral and steroid therapy, visual acuity was $20 / 40$ and the corneal lesion was recovered except corneal opacity (Fig. 1B). No recurrence happened for 1 year.

Generally, immune status, trauma, heat, and emotional stress are known as the cause of recurrence in HSV infections [2]. In our case, thermal stress was estimated to have affected HSV reactivation based on two points. At first, right eye that has already had history of recurrence and thought to be vulnerable to reactivation was the only one to recur although the device was applied on both eyes. Secondly, the lesion was occurred right after the use of thermal device. There are reports that heat stress plays an important role in reactivation of HSV. Halford et al. [3] demonstrated reactivation of HSV was related to heat stress duration. The ganglion cells were incubated in a thermostat at $43^{\circ} \mathrm{C}$ in experiment group and normal environment in control group, and were compared by the reverse transcription polymerase chain reaction. In the experiment group, infectious virus appeared in $75 \%$ after thermal stress [3]. Another study reported the latent infection with type I HSV in mice placed in water at $43^{\circ} \mathrm{C}$ for 10 minutes, and reactivation of the HSV was observed in the cornea [4]. The device used in this case had a laser irradiation function with a wavelength of $650 \mathrm{~nm}$ and a thermal function at $45^{\circ} \mathrm{C}$. Since $650 \mathrm{~nm}$ is known to have no significant change to cornea, the lesion does not seem to be affected by laser function and thermal function applied to the eye may trigger the reactivation of virus remained in the trigeminal ganglion and induce the recurrence of keratitis [5]. 


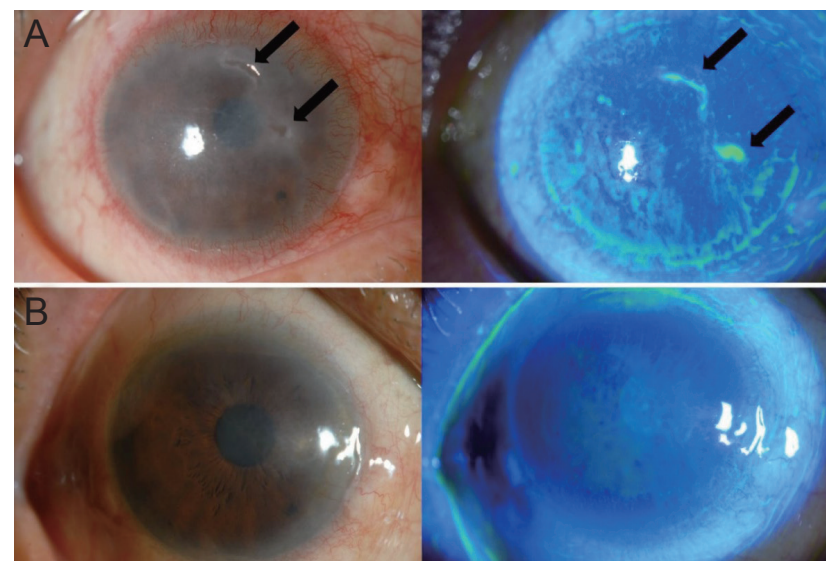

Fig. 1. Anterior segment photographs after the use of thermal therapy device. (A) On the first day of recurrence, corneal dendritic lesions (arrows), corneal edema, keratic precipitate, and stromal infiltration were observed. (B) After 3 months of treatment, only corneal opacity was remained. The patient provided written informed consent for publication of the research details and clinical images.

Although limitations of this case are the evidence of the herpetic keratitis recurrence induced by $36^{\circ} \mathrm{C}$ to $45^{\circ} \mathrm{C}$ indirect thermal stress has not been fully provided, and only one patient report and further review of the feasibility of such case collection will be needed, patients with a history of herpetic keratitis should be cautious about the recurrence when using the thermal medical device because heat stress may reactivate the virus.

\section{Min Seung Kang, Sang Min Lee, Ji Eun Lee} Department of Ophthalmology, Pusan National University Yangsan Hospital, Pusan National University School of Medicine, Yangsan, Korea

Research Institute for Convergence of Biomedical Science and Technology, Pusan National University Yangsan Hospital, Pusan National University School of Medicine, Yangsan, Korea E-mail (JiEunLee): jiel75@pusan.ac.kr

\section{Sung Il Kim}

Department of Ophthalmology, Pusan National University Yangsan Hospital, Pusan National University School of Medicine, Yangsan, Korea ABC Eye Clinic, Busan, Korea

\section{Conflicts of Interest}

No potential conflict of interest relevant to this article was reported.

\section{Acknowledgements}

None.

\section{Funding}

This work was supported by Pusan National University Research Grant, 2020.

\section{References}

1. Lobo AM, Agelidis AM, Shukla D. Pathogenesis of herpes simplex keratitis: the host cell response and ocular surface sequelae to infection and inflammation. Ocul Surf 2019;17:40-9.

2. Yan C, Luo Z, Li W, et al. Disturbed Yin-Yang balance: stress increases the susceptibility to primary and recurrent infections of herpes simplex virus type 1. Acta Pharm Sin B 2020;10:383-98.

3. Halford WP, Gebhardt BM, Carr DJ. Mechanisms of herpes simplex virus type 1 reactivation. J Virol 1996;70:505160.

4. Gebhardt BM, Kaufman HE, Hill JM. Effect of acyclovir on thermal stress-induced herpesvirus reactivation. Curr Eye Res 2004;29:137-44.

5. Lee HS, Cui L, Li Y, et al. Influence of light emitting diode-derived blue light overexposure on mouse ocular surface. PLoS One 2016;11:e0161041. 